\title{
A NOTE ON HOLOMORPHIC IMBEDDINGS OF THE CLASSICAL CARTAN DOMAINS INTO THE UNIT BALL
}

\author{
YOSHIHISA KUBOTA
}

\begin{abstract}
Let $D$ be a classical Cartan domain and let $B$ be the unit ball. We find the exact value of the supremum of the set of positive numbers $\rho$ satisfying the condition: $\rho B \subset f(D)$ for a certain holomorphic imbedding $f: D \rightarrow B$.
\end{abstract}

1. This paper is concerned with the following problem: Let $D$ be a bounded homogeneous domain in $\mathbf{C}^{n}$. By $\delta(D)$ we denote the family of holomorphic imbeddings $f$ of $D$ into the unit ball $B_{n}$ in $C^{n}$ such that $f(D) \ni 0$. For each $f \in \mathcal{S}(D)$ we define

$$
\rho_{f}=\sup \left\{\rho>0: \rho B_{n} \subset f(D)\right\}
$$

where $\rho B_{n}=\left\{\rho z: z \in B_{n}\right\}$. Further we define

$$
\rho(D)=\sup \left\{\rho_{f}: f \in S(D)\right\} .
$$

Obviously $\rho(D)$ is invariant under biholomorphic mappings. It is required to find the exact value of $\rho(D)$. Alexander [1] proved that for the unit polydisc $U$ in $\mathbf{C}^{n}$, $\rho(U)=n^{-1 / 2}$.

In this paper we note that by appealing to the method of Alexander we are able to find the values $\rho(D)$ for the classical Cartan domains.

By a classical Cartan domain we understand a domain of one of the following four types:

$$
\begin{aligned}
& R_{\mathrm{I}}(r, s)=\left\{Z=\left(z_{j k}\right): I-Z \bar{Z}^{\prime}>0, \text { where } Z \text { is an } r \times s \text { matrix }\right\}(r \leqslant s), \\
& R_{\mathrm{II}}(p)=\left\{Z=\left(z_{j k}\right): I-Z \bar{Z}^{\prime}>0, \text { where } Z \text { is a symmetric matrix of order } p\right\}, \\
& R_{\mathrm{III}}(q)=\left\{Z=\left(z_{j k}\right): I-Z \bar{Z}^{\prime}>0, \text { where } Z\right. \text { is a skew-symmetric matrix of order } \\
& R_{\mathrm{IV}}(n)=\left\{z=\left(z_{1}, \ldots, z_{n}\right): 1+\left|z z^{\prime}\right|^{2}-2 z \bar{z}^{\prime}>0,1-\left|z z^{\prime}\right|>0\right\} .
\end{aligned}
$$
$q\}$,

Here $I$ is the identity matrix and $\bar{Z}$ denotes the conjugate matrix of $Z$ and $Z^{\prime}$ the transposed matrix of $Z$. The complex dimensions of these four domains are $r s$, $p(p+1) / 2, q(q-1) / 2, n$, respectively.

We shall prove the following theorems.

Received by the editors April 16, 1980.

1980 Mathematics Subject Classification. Primary 32 H99.

Key words and phrases. Classical Cartan domain, holomorphic imbedding, bounded homogeneous domain, biholomorphic mapping. 
THEOREM 1.

(1.1) $\rho\left(R_{\mathrm{I}}(r, s)\right)=r^{-1 / 2}$,

(1.2) $\rho\left(R_{\mathrm{II}}(p)\right)=p^{-1 / 2}$,

(1.3) $\rho\left(R_{\mathrm{III}}(q)\right)=[q / 2]^{-1 / 2}$,

where $[q / 2]$ denotes the integral part of $q / 2$,

(1.4) $\rho\left(R_{\mathrm{IV}}(n)\right)=2^{-1 / 2}$.

TheOREM 2. If $D_{1}, \ldots, D_{m}$ are classical Cartan domains, then

$$
\rho\left(D_{1} \times \cdots \times D_{m}\right)=\left[\rho\left(D_{1}\right)^{-2}+\cdots+\rho\left(D_{m}\right)^{-2}\right]^{-1 / 2} .
$$

2. We begin with two lemmas. By applying the method of Alexander [1] we are able to prove the following lemma. For the sake of completeness we give a proof.

LEMMA 1. Let $D$ be a bounded homogeneous domain in $\mathbf{C}^{n}$ satisfying the conditions:

$$
\left\{z=\left(z_{1}, \ldots, z_{n}\right):\left|z_{\alpha_{j}}\right|<1 \text { for } j=1, \ldots, m \text { and } z_{\alpha}=0 \text { for the other } \alpha \text { 's }\right\} \subset D \text {, }
$$

where $1 \leqslant \alpha_{1}<\cdots<\alpha_{m} \leqslant n$,

(2) for each $j(1 \leqslant j \leqslant m)$

$$
\left\{z=\left(z_{1}, \ldots, z_{n}\right):\left|z_{\alpha_{j}}\right|=1 \text { and } z_{\alpha}=0 \text { for the other } \alpha \text { 's }\right\} \subset \partial D .
$$

If $f \in \mathcal{S}(D)$ and $\rho B_{n} \subset f(D)$, then $\rho \leqslant m^{-1 / 2}$.

Proof. Let $f=\left(f_{1}, \ldots, f_{n}\right)$ be a mapping in $\delta(D)$. Since $D$ is homogeneous, we may assume that $f(0)=0$. We set

$$
g_{\alpha}\left(\zeta_{1}, \ldots, \zeta_{m}\right)=f_{\alpha}\left(0, \ldots, \zeta_{1}, \ldots, \zeta_{m}, \ldots, 0\right) \quad(\alpha=1, \ldots, n),
$$

where $\zeta_{j}$ is in the $\alpha_{j}$ th position in the right-hand side. The function $g_{\alpha}$ is holomorphic in the polydisc $U=\left\{\zeta=\left(\zeta_{1}, \ldots, \zeta_{m}\right):\left|\zeta_{j}\right|<1, j=1, \ldots, m\right\}$. Hence $g_{\alpha}$ admits an expansion

$$
g_{\alpha}(\zeta)=\sum a_{v_{1} \cdots v_{m}}^{(\alpha)} \zeta_{1}^{v_{1}} \cdots \zeta_{m}^{v_{m}}, \quad a_{0}^{(\alpha)} \cdots_{0}=0
$$

which converges uniformly on compact subsets of $U$. Firstly, by condition (1) we have

$$
\begin{aligned}
& \sum_{\alpha=1}^{n}\left(\sum\left|a_{v_{1} \cdots v_{m}}^{(\alpha)}\right|^{2}\right) \\
& \quad=\lim _{r \rightarrow 1} \frac{1}{(2 \pi)^{m}} \int_{0}^{2 \pi} \cdots \int_{0}^{2 \pi}\left\{\sum_{\alpha=1}^{n}\left|g_{\alpha}\left(r e^{i \theta_{1}}, \ldots, r e^{i \theta_{m}}\right)\right|^{2}\right\} d \theta_{1} \cdots d \theta_{m} \\
& \leqslant 1 .
\end{aligned}
$$

On the other hand, by condition (2), we have

$$
\begin{aligned}
\rho^{2} & \leqslant \frac{1}{2 \pi} \int_{0}^{2 \pi}\left\{\sum_{\alpha=1}^{n}\left|g_{\alpha}\left(0, \ldots, e^{i \theta_{j}}, \ldots, 0\right)\right|^{2}\right\} d \theta_{j} \\
& =\sum_{\alpha=1}^{n}\left(\sum\left|a_{0}^{(\alpha)} \cdots v_{j} \cdots 0\right|^{2}\right),
\end{aligned}
$$


where $g_{\alpha}\left(0, \ldots, e^{i \theta_{j}}, \ldots, 0\right)$ is the radial limit

$$
\lim _{r \rightarrow 1} g_{\alpha}\left(0, \ldots, r e^{i \theta_{j}}, \ldots, 0\right)
$$

which exists a.e. on $\left\{\theta_{j}: 0 \leqslant \theta_{j} \leqslant 2 \pi\right\}$. Therefore we obtain $m \rho^{2} \leqslant 1$.

Next, instead of $R_{\text {II }}(p)$ we consider the following modified domain:

$\hat{R}_{\mathrm{II}}(p)=\left\{Z=\left(z_{j k}\right): z_{j k}=\sqrt{2} x_{j k}(j \neq k), z_{j j}=x_{j j}\right.$, where $\left.X=\left(x_{j k}\right) \in R_{\mathrm{II}}(p)\right\}$.

Further, instead of $R_{\mathrm{IV}}(n)$ we consider the following domain:

$$
\begin{aligned}
R_{\mathrm{IV}}^{*}(n)=\{ & z=\left(z_{1}, \ldots, z_{n}\right): 1+\left|z_{1} z_{2}+\frac{1}{2}\left(z_{3}^{2}+\cdots+z_{n}^{2}\right)\right|^{2} \\
& \left.-\left(\left|z_{1}\right|^{2}+\cdots+\left|z_{n}\right|^{2}\right)>0,1-\left|z_{1} z_{2}+\frac{1}{2}\left(z_{3}^{2}+\cdots+z_{n}^{2}\right)\right|>0\right\} .
\end{aligned}
$$

The domain $R_{\mathrm{IV}}^{*}(n)$ is obtained from $R_{\mathrm{IV}}(n)$ by the biholomorphic mapping $\varphi=\left(\varphi_{1}, \ldots, \varphi_{n}\right), \varphi_{1}(z)=z_{1}+i z_{2}, \varphi_{2}(z)=z_{1}-i z_{2}, \varphi_{\alpha}(z)=\sqrt{2} z_{\alpha}(\alpha=3, \ldots, n)$.

We have the following:

\section{LEMMA 2.}

$$
\begin{aligned}
& B_{n} \subset R_{\mathrm{I}}(r, s) \subset \sqrt{r} B_{n} \quad(n=r s), \\
& B_{n} \subset \hat{R}_{\mathrm{II}}(p) \subset \sqrt{p} B_{n} \quad(n=p(p+1) / 2), \\
& B_{n} \subset R_{\mathrm{III}}(q) \subset \sqrt{[q / 2]} B_{n} \quad(n=q(q-1) / 2), \\
& B_{n} \subset R_{\mathrm{IV}}^{*}(n) \subset \sqrt{2} B_{n} .
\end{aligned}
$$

Proof. In [4] we showed that

$$
R_{\mathrm{I}}(r, s) \subset \sqrt{r} B_{n}, \quad \hat{R}_{\mathrm{II}}(p) \subset \sqrt{p} B_{n}, \quad R_{\mathrm{III}}(q) \subset \sqrt{[q / 2]} B_{n} .
$$

To show that $B_{n} \subset R_{\mathrm{I}}(r, s)$, let $\lambda_{1}, \ldots, \lambda_{r}$ be the characteristic roots of $Z \overline{Z^{\prime}}$, where $Z$ is an $r \times s$ matrix in $B_{n}$. Then we have

$$
\lambda_{1}+\cdots+\lambda_{r}=\operatorname{trace} Z \bar{Z}^{\prime}=\sum_{j=1}^{r} \sum_{k=1}^{s}\left|z_{j k}\right|^{2}<1
$$

and $\lambda_{j} \geqslant 0(j=1, \ldots, r)$; therefore $\lambda_{j}<1$. This implies that $B_{n} \subset R_{\mathrm{I}}(r, s)$.

Similarly we obtain $B_{n} \subset \hat{R}_{\mathrm{II}}(p)$. If $Z$ is a skew-symmetric matrix, the characteristic roots of $Z \bar{Z}^{\prime}$ are double roots or equal to 0 [2]. Hence we have $B_{n} \subset R_{\text {III }}(q)$. The last relation $B_{n} \subset R_{\mathrm{IV}}^{*}(n) \subset \sqrt{2} B_{n}$ is obvious.

3. We now turn to the proof of theorems. We consider an $r \times s$ matrix $Z$ such that

$$
Z=\left(\begin{array}{ccccccc}
\zeta_{1} & 0 & \cdots & 0 & 0 & \cdots & 0 \\
0 & \zeta_{2} & \cdots & 0 & 0 & \cdots & 0 \\
\cdots & \cdots & \cdots & \cdots & \cdots & \cdots & 0 \\
0 & 0 & \cdots & \zeta_{r} & 0 & \cdots & 0
\end{array}\right)
$$

If $\left|\zeta_{j}\right|<1(j=1, \ldots, r)$, then $Z$ belongs to $R_{\mathrm{I}}(r, s)$. Further if $\left|\zeta_{k}\right|=1$ and $\zeta_{j}=0$ $(j \neq k)$, then $Z$ belongs to $\partial R_{\mathrm{I}}(r, s)$. Therefore (1.1) is an immediate consequence of Lemmas 1 and 2. Similarly we obtain $\rho\left(\hat{R}_{\mathrm{II}}(p)\right)=p^{-1 / 2}$. 
Considering a skew-symmetric matrix $Z$ of order $q$ such that

$$
Z=\left(\begin{array}{cc}
0 & \zeta_{1} \\
-\zeta_{1} & 0
\end{array}\right)+\cdots+\left(\begin{array}{cc}
0 & \zeta_{m} \\
-\zeta_{m} & 0
\end{array}\right) \quad(q=2 m)
$$

or

$$
Z=\left(\begin{array}{cc}
0 & \zeta_{1} \\
-\zeta_{1} & 0
\end{array}\right)+\cdots+\left(\begin{array}{cc}
0 & \zeta_{m} \\
-\zeta_{m} & 0
\end{array}\right)+0 \quad(q=2 m+1),
$$

we obtain (1.3). To prove (1.4) we consider a point $z$ in $\mathbf{C}^{n}$ of the form $z=\left(\zeta_{1}, \zeta_{2}\right.$, $0, \ldots, 0)$, Then we have

$$
\rho\left(R_{\mathrm{IV}}^{*}(n)\right)=2^{-1 / 2} \text {. }
$$

This completes the proof of Theorem 1 .

Theorem 2 follows from Lemmas 1, 2 and the following fact: if $D_{1}, \ldots, D_{m}$ are domains satisfying

$$
B_{n_{v}} \subset D_{v} \subset \rho_{v} B_{n_{v}} \quad(v=1, \ldots, m)
$$

then

$$
B_{n} \subset D_{1} \times \cdots \times D_{m} \subset \sqrt{\rho_{1}^{2}+\cdots+\rho_{m}^{2}} B_{n} \quad\left(n=n_{1}+\cdots+n_{m}\right) .
$$

\section{REFERENCES}

1. H. Alexander, Extremal holomorphic imbeddings between the ball and polydisc, Proc. Amer. Math. Soc. 68 (1978), 200-202.

2. L. K. Hua, On the theory of automorphic functions of a matrix variable. I. Geometrical basis, Amer. J. Math. 66 (1944), 470-488.

3. Harmonic analysis of functions of several complex variables in the classical domains, Transl. Math. Monographs, vol. 6, Amer. Math. Soc., Providence, R. I., 1963.

4. Y. Kubota, An extremal problem on the classical Cartan domains, Kodai Math. J. 4 (1981), 278-287.

5. K. H. Look, Schwarz lemma and analytic invariants, Sci. Sinica 7 (1958), 453-504.

Department of Mathematics, Tokyo Gakugei University, Koganei-ShI, Tokyo 184, Japan 